University of Wollongong

Research Online

Faculty of Engineering - Papers (Archive)

Faculty of Engineering and Information

Sciences

$1-1-2005$

\title{
Modeling the effect of p53 on tumor heterogeneity and the mutator phenotype
}

Melissa Ryan

University of Adelaide

Matthew J. Berryman

University of Wollongong, mberryma@uow.edu.au

Derek Abbott

University of Adelaide, dabbott@eleceng.adelaide.edu.au

Follow this and additional works at: https://ro.uow.edu.au/engpapers

Part of the Engineering Commons

https://ro.uow.edu.au/engpapers/4905

\section{Recommended Citation}

Ryan, Melissa; Berryman, Matthew J.; and Abbott, Derek: Modeling the effect of p53 on tumor heterogeneity and the mutator phenotype 2005, 144-152.

https://ro.uow.edu.au/engpapers/4905 


\title{
Modeling the effect of p53 on tumor heterogeneity and the mutator phenotype
}

\author{
Melissa Ryan ${ }^{a},{ }^{b}$, Matthew J. Berryman ${ }^{a}$, and Derek Abbott ${ }^{a}$ \\ ${ }^{a}$ Centre for Biomedical Engineering and \\ School of Electrical and Electronic Engineering, \\ The University of Adelaide, SA 5005, Australia. \\ ${ }^{b}$ School of Informatics and Engineering, Flinders University, Adelaide, SA 5042, Australia
}

\begin{abstract}
p53 is an important gene, involved in apoptosis (programmed cell death), DNA repair, and cell cycle progression. We explore the selective advantages and disadvantages of mutations in the p53 gene on tumor cells, and the heterogeneity of tumor cell populations. Based on an evolutionary computational approach, our model considers changes in mutation rate caused by lack of DNA repair processes, and the lack of apoptosis caused by mutations in p53. We find that the degree of robustness of p53 to mutations has a significant effect on the tumor heterogeneity and "fitness", with clinical consequences for people who inherit p53 mutations.
\end{abstract}

Keywords: Evolutionary computation, genetic algorithms, cancer, mutation

\section{INTRODUCTION}

One in three people are affected by cancer in their lifetime. ${ }^{1}$ Cancer is caused by multiple DNA mutations that allow cells to proliferate without limit. ${ }^{2}$ The p53 protein is a potent tumor suppressor, and plays a major role in maintaining a healthy cell cycle. During the cell cycle, however, DNA inside cells experiences spontaneous or environmentally induced mutations. Mutations occurring in vital parts of the DNA sequence can be deleterious for the cell.

One type of mutation that can alter DNA sequences is base pair substitution mutations. ${ }^{3}$ As the name suggests, base pair substitution mutations occur when one of the nucleotide bases is changed; this change is classified as either a transition or a transversion. A transition occurs when a purine (A or $\mathrm{G}$ ) is changed to a purine or a pyrimidine ( $\mathrm{C}$ or $\mathrm{T}$, or $\mathrm{U}$ in $\mathrm{RNA}$ ) to a pyrimidine. For example, consider a base pairing of guanine (a purine) and cytosine (a pyrimidine). If during translation the guanine becomes adenine (another purine), the result is the base pairing of adenine and cytosine in the new strand of DNA. If instead, the guanine had been changed to cytosine or thymine (or uracil in RNA), the result would be a base pairing of two pyrimidine's, which is genomically unstable. This type of mutation, when a purine is changed to a pyrimidine or a pyrimidine to a purine, is known as a transversion. ${ }^{4}$ Because of the speed at which transcription occurs, there is a large window of opportunity for errors to occur. Some mutations, however, can be silent $(i)$ if the mutation results in a change of the codon, but not the amino acid it codes for, or (ii) if it alters the amino acid, but not the protein. Conversely missense mutations alter the genes such that the amino acid coded for is changed, or codons that originally coded for amino acids are transformed into stop codons and vice-versa. ${ }^{3}$ Mutations to either the p53 gene itself, or the pathways activated by p53 are the single most common mutations found in cancer and have been implicated in over 50 per cent of cancer cases. ${ }^{5,6}$ It is a mutation of this gene or its effector pathways that consequently results in malignant tumours. ${ }^{7}$

In healthy cells, the p53 protein is present at very low concentrations, in an inactive form. Levels of p53 in the cell are maintained by a negative feedback loop between it and another protein Mdm2. ${ }^{8,9}$ There are several checkpoints in the cell cycle which can prevent the cell from progressing if DNA damage is detected. Activation of the p53 protein can occur at two of these checkpoints in the cell cycle. If DNA damage is detected, p53 is

Send correspondence to Derek Abbott

E-mail: dabbott@eleceng.adelaide.edu.au, Telephone: +61 883035748 
indirectly activated and stops cells in the G1 and G2 phase from progressing, slows down cells in the S phase and induces transcription of repair genes. ${ }^{10}$ Such forms of DNA damage that have been shown to activate p53 include those caused by ultraviolet (UV) light, other ionizing radiation, and exposure to radio-mimetic drugs. ${ }^{11}$ Activation of p53 in response to DNA damage increases the ability of the p53 protein to bind to DNA ${ }^{11}$ and causes a decrease in its affinity for Mdm2 and hence a decrease in degradation of itself. ${ }^{12}$ Simultaneously, the p53 protein induces apoptosis and initiates transcription of proteins involved in DNA repair and cell cycle arrest. ${ }^{6}$ Cell cycle arrest restricts the cell from entering into the next phase in the cell cycle, until the DNA damage has been repaired. ${ }^{12}$ If the DNA damage is too severe, then the cell will undergo programmed cell death, known as apoptosis. This can be mediated either, by p53 initiating transcription of proteins such as the Bax protein; or directly, by stimulation of the mitochondria to produce excess toxic reactive oxygen species. ${ }^{8}$

Table 1 identifies some of the mutations that can occur and their affects on the activities of p53.

Table 1. From Vogelstein et al., ${ }^{8}$ this table shows the effect of mutations on p53 and the many ways it may malfunction in tumors.

\begin{tabular}{|l|l|l|}
\hline $\begin{array}{l}\text { Mechanism of inactivating } \\
\text { p53 }\end{array}$ & Typical tumors & Effect of inactivation \\
\hline \hline $\begin{array}{l}\text { Amino-acid-changing mutation } \\
\text { in the DNA-binding domain }\end{array}$ & $\begin{array}{l}\text { Colon, breast, lung, bladder, } \\
\text { brain, pancreas, stomach, } \\
\text { esophagus and many others }\end{array}$ & $\begin{array}{l}\text { Prevents p53 from binding to } \\
\text { specific DNA sequences and ac- } \\
\text { tivating the adjacent genes }\end{array}$ \\
\hline $\begin{array}{l}\text { Deletion of the carboxy- } \\
\text { terminal domain }\end{array}$ & $\begin{array}{l}\text { Occasional tumors at many dif- } \\
\text { ferent sites }\end{array}$ & $\begin{array}{l}\text { Prevents the formation of } \\
\text { tetramers of p53 }\end{array}$ \\
\hline $\begin{array}{l}\text { Multiplication of the Mdm2 } \\
\text { gene in the genome }\end{array}$ & Sarcomas, brain & $\begin{array}{l}\text { Extra Mdm2 stimulates the } \\
\text { degradation of p53 }\end{array}$ \\
\hline $\begin{array}{l}\text { Viral infection } \\
\text { Cervix, liver, lymphomas }\end{array}$ & $\begin{array}{l}\text { Products of viral oncogenes bind } \\
\text { to and inactivate p53 in the cell, } \\
\text { in some cases stimulating p53 } \\
\text { degradation }\end{array}$ \\
\hline $\begin{array}{l}\text { Deletion of the p14 }{ }^{\mathrm{ARF}} \text { gene } \\
\text { Mislocalization of p53 to the cy- } \\
\text { toplasm, outside the nucleus }\end{array}$ & $\begin{array}{l}\text { Breast, neuroblastomas } \\
\text { especially when p53 itself is not } \\
\text { mutated }\end{array}$ & $\begin{array}{l}\text { Failure to inhibit Mdm2 and } \\
\text { trol }\end{array}$ \\
\hline
\end{tabular}

DNA is the only macromolecule in the body that is repaired by cells. ${ }^{3}$ Eukaryotic repair mechanisms play a major role in maintaining the integrity of the genome. ${ }^{4}$ In cells, DNA repair is constantly occurring and is essential for their survival. DNA damage reversal is the simplest form of repair; enzymatic action by DNA ligase repairs simple breaks in one strand of the DNA. ${ }^{3}$ Another form of DNA repair is damage removal, of which there are three distinct forms; base excision repair (BER), nucleotide excision repair (NER) and mismatch repair (MMR). ${ }^{13}$ Although not truly a repair mechanism, cells can also develop ways of coping with the damage (damage tolerance). ${ }^{3}$

Evidence, from both mouse and human models, has shown direct or indirect involvement of p53 in nucleotide excision repair. ${ }^{14,15}$ Lesions that distort the double helix, such as a T-dimer, are repaired by NER. ${ }^{4}$ NER involves the excision of an oligonucleotide; this comprises the breaking of phosphodiester bonds, on the same strand, on either side of the lesion; the subsequent gap is filled DNA ligase and repair synthesis. ${ }^{16}$

When p53 function is reduced due to mutations, the DNA repair capabilities of the cell are reduced. ${ }^{2}$ The cell is unable to cease cell cycle progression at DNA checkpoints and perform DNA repair, so the cell will continue through the cell cycle and the daughter cells will receive mutated DNA, or incomplete or broken sets of chromosomes. ${ }^{12}$ Ordinarily, DNA damage, or telomere malfunction would trigger apoptosis through p53 to remove these cells, ${ }^{17}$ however mutations to p53 or its effectors can prevent programmed cell death occurring. The lack of apoptosis causes uncontrolled cell proliferation despite DNA damage and has been shown to promote 
oncogenic transformations and tumor development in mouse model systems. ${ }^{2,17}$ Attardi and Jacks performed studies on both homozygous and heterozygous mice for a deletion in the p53 gene. ${ }^{18}$ Their results showed that both groups of mice developed tumors at a high frequency; however the homozygotes had a significantly shorter average latency for the tumors and shorter life span. Their studies of differences between the two sets of mice also indicated that the affects of p53 inhibition vary in different tissue types.

The revelation that checkpoint function has been strongly implicated in the prevention of cancer, ${ }^{10}$ has provided significant breakthroughs in research to developing alternative cancer treatments. Such treatments include the reintroduction of wild type p53 to p53 mutant tumor lines, which has been shown to induce apoptosis and tumor regression, when performed in conjunction with chemotherapy. ${ }^{17}$ Other suggested therapeutic strategies involving p53 include the restoration of p53 function to mutant p53 tumors by p53 gene therapy; also, drugs that are able to mimic the effects of p53 or modify its effector pathways may useful. ${ }^{7}$ The p53 has also been suggested as a diagnostic tool for physicians. Polymerase chain reaction (PCR)-based techniques have been used to detect mutant p53 in exfoliated cells in bladder and lung cancers. ${ }^{7}$ This would be a useful strategy in early detection of cancers, or identifying potential cancer patients.

Here we look at the effects of mutation and repair rates and apoptosis on a population of cells through a number of generations. We propose a model that represents a population of cells of given genome length, initially set with a random number of mutations, that reproduce by asexual recombination. The behavior of p53 is modeled by considering part of the genome to be a p53 gene, with two separate regions: one governing apoptosis, the other governing repair of mutations. We consider different mutation rates, repair rates, the number of mutations before apoptosis occurs, and the number of mutations before each region of p53 is significantly affected. The results on the growth and heterogeneity of tumor populations are determined, with implications for both when p53 mutations should be detected, and for treatment of p53.

\section{METHODS}

A binary string of given length, $G$, was used to represent each individual; each bit could be interpreted as being an allele or a base pair etc. A "0" was defined as being a healthy bit/allelle and a "1" a mutated bit/allelle. The phenotype of each genome was expressed as the number of mutations it contained (ie. the sum of " 1 " bits):

$$
I_{x}=\text { displaystyle } \sum_{i=1}^{G} x_{i}
$$

where $x_{i}$ is the $i^{\text {th }}$ bit of the string.

The fitness function for each individual was simply the sum of " 0 " bits and could be expressed in terms of $I_{x}$, as:

$$
F(x)=G-I_{x}
$$

The initial progeny was randomly created as an $N$ by $G$ array, where each row of the array represented an individual, giving an initial population size $N$. Each bit of the array was set with a 90 percent probability of being healthy and 10 percent probability of being mutated.

A portion, $g$, of each genome was used to represent the p53 gene. The size of $g$ was set with respect to $G$. As discussed previously, the p53 gene has numerous roles in maintaining a healthy cell cycle, such as cell cycle arrest, apoptosis and DNA repair. ${ }^{8}$ In our model, the region representing the p53 gene was designed such that 60 percent coded for apoptosis, $g_{1}$, and the other 40 percent for DNA repair, $g_{2}$.

The evolution of the progeny was studied over fifty cell cycles/generations. During each cell cycle, all individuals underwent conditional spontaneous mutation, DNA repair and apoptosis.

Our model considers both silent and missense base pair substitution mutations. Cells were mutated with mutation rate $m$. The rate of spontaneous mutations for $E$. coli was stated by Elliott to be 1 error in every $10^{8}$ nucleotides replicated. ${ }^{4}$ Other rates of mutation have been found to be in the order of 1 nucleotide change per $10^{9}$ nucleotides per cell generation for other bacteria ${ }^{12}$ and somewhere in the order of 1 in $10^{6}$ or $10^{5}$ nucleotides replicated for humans. ${ }^{4}$ Since our model considers only genomes of one hundred bits and the number of 
generations it considers is limited due to the time required to perform the computations, using mutation rates of $1 / 10^{6}$ over a limited number of generations would produce little or no effect on the population. To compensate for this, much larger mutation rates, in the range of 0.05 to 0.50 , were used.

For individuals in our model to be eligible for DNA repair, a minimum fitness was required of the $g_{2}$ region. Each individual that satisfied this minimum fitness level underwent DNA repair, with a repair rate $m_{1}$. Again, this rate had no reflection on the actual rate of repair occurring in cells. The range of values used for $m_{1}$ was the same as those used for $m$. Various combinations of $m$ and $m_{1}$ were used, such as $m_{1}=m, m_{1}=2 m, m_{1}=10 m$, etc. When cells underwent DNA mutation or repair, each bit/allelle in its genome had an equal likelihood of experiencing a mutation.

If an individual did not meet the minimum fitness requirement then apoptosis of the cell was required. For apoptosis to occur, however, a minimum fitness of the $g_{1}$ region was also essential. Individuals, which required apoptosis (eg. $F(x)<f$ ) and that had a $g_{1}$ region with minimum fitness (eg. $\left.F\left(g_{1}\right)>p_{1}\right)$, were then selected for apoptosis. If the $g_{1}$ region was too mutated, then apoptosis would not occur and the severely mutated cell would survive and could then undergo cell division to produce two daughter cells with extremely mutated genomes.

Cell division occurred at the end of each cell cycle, so that each successive generation had twice the population size of its parent generation. To ensure the cells did not proliferate out of control, random individuals were killed off directly after cell division so that the population size was kept to a maximum of ten thousand.

\section{RESULTS}

The genome size was set to $G=100$, with $g=8$ and the initial population size set to $N=50$. For cells to survive, it is essential that the rate of repair exceed the rate of mutation, so initially the mutation rate was kept constant at $m=0.05$ and the following repair rates were used: $m_{1}=0.05 ; m_{1}=0.1 ; m_{1}=0.2$ and $m_{1}=0.5$. Due to the random element within our model, ten trials were run for each of these repair rates, recording the mean phenotype (number of mutations) of the progeny at each generation, and the average of each was used.

The mean phenotype $\bar{I}_{x}(t)$ was then plotted against time $t$ (in cell cycles), for each repair rate considered, and the resultant plot in Figure 1 shows a second order polynomic relationship between the mean phenotype $\bar{I}_{x}(t)$ and time $t$.

From these plots, we see that the phenotype of the population continues to increase with time, for all the repair rates. In other words, if we continued to observe the genomic activity of the cells, all the cells in the progeny would eventually acquire a phenotype of 100 (i.e. 100 mutations); the difference between the curves, however, is the rate of mutation acquisition. The latency to reach this level of mutation would be different for the different repair rates.

Differentiating $\bar{I}_{x}(t)$ with respect to $t$ we see the rate of mutation acquisition per cell division/generation. Table 2 shows the mean phenotype $\bar{I}_{x}(t)$ and its derivative $\bar{I}_{x}(t)^{\prime}$ for each curve in Figure 1 .

Table 2. Mean phenotype $\bar{I}_{x}(t)$ and its derivative $\bar{I}_{x}(t)^{\prime}$ for various repair rates (mutation rate $=0.05$ )

\begin{tabular}{|c|c|c|}
\hline Repair rate & $I_{x}(t)$ & $I_{x}(t)^{\prime}$ \\
\hline \hline 0.05 & $-0.0173 t^{2}+2.3359 t+12.118\left(R^{2}=0.997\right)$ & $-0.346 t+2.3359$ \\
\hline 0.1 & $-0.0074 t^{2}+1.6155 t+12.703\left(R^{2}=0.9962\right)$ & $-0.148 t+1.6155$ \\
\hline 0.2 & $0.0018 t^{2}+0.8957 t+11.206\left(R^{2}=0.9986\right)$ & $0.0036 t+0.8957$ \\
\hline 0.5 & $0.0052 t^{2}+0.3179 t+6.187\left(R^{2}=0.9943\right)$ & $0.0104 t+0.3179$ \\
\hline
\end{tabular}

Plotting the derivative $\bar{I}_{x}(t)^{\prime}$ for each mutation rate in Figure 2, we see that the curves for $m_{1}=0.05$ and $m_{1}=0.1$ both have a decreasing mutation acquisition rate, but the curves for $m_{1}=0.2$ and $m_{1}=0.5$ both have an increasing rate of mutation acquisition.

From these results, we hypothesize that the rate of mutation acquisition is of the form: $a x^{2}+b x+c$ (and concave $\therefore a<0$ ). Integrating this back with respect to $t$, to give the expression for the mean phenotype, would infer that the curves representing the behavior of the phenotype over time, are in fact of the form: 


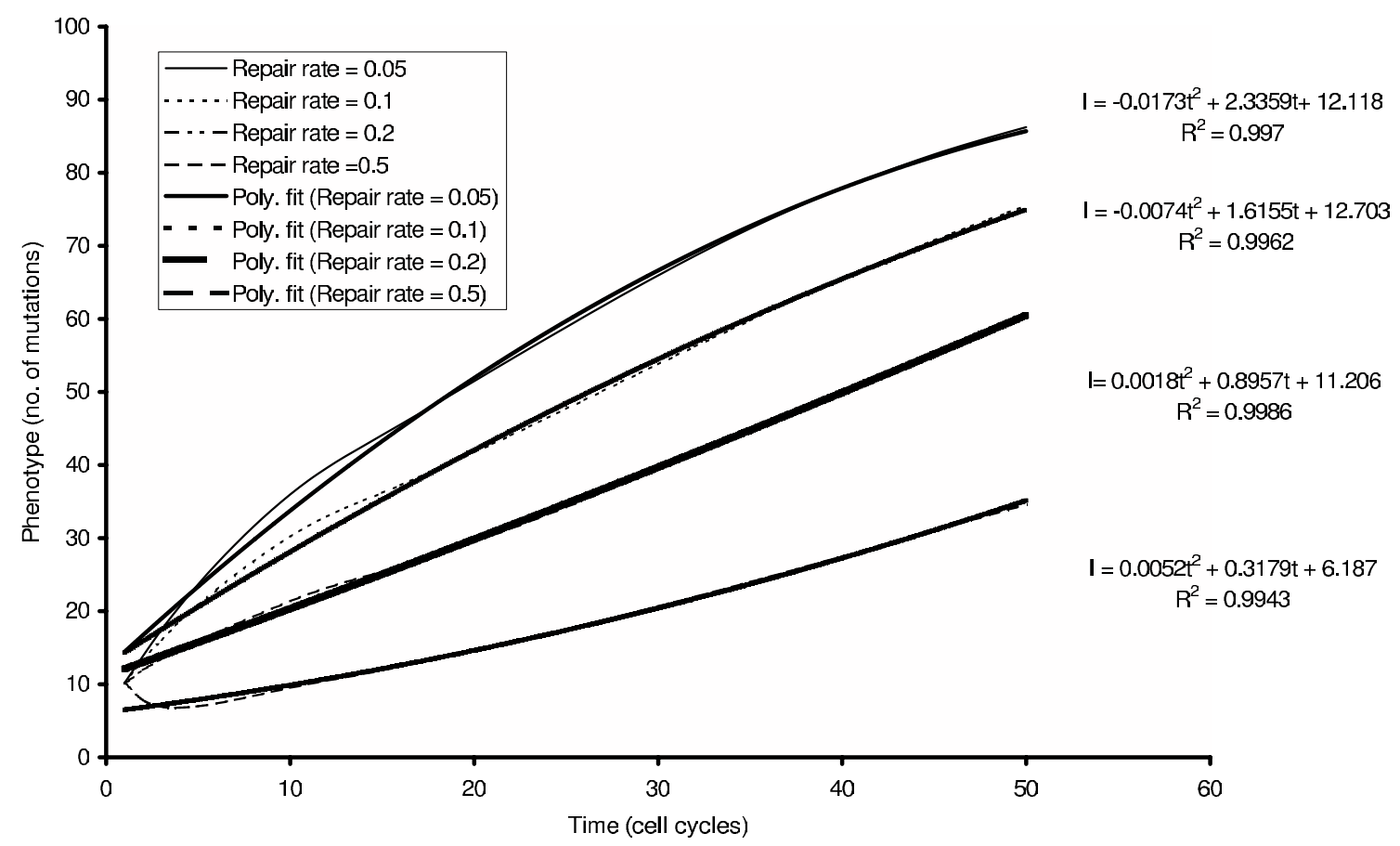

Figure 1. Mean phenotype (number of mutations) of cells against generations. Mutation rate $m=0.05$. Repair rates considered: $m=0.05 m=0.1 m=0.2$ and $m=0.5$. The actual curves are represented by the four different line types. A cubic polynomial was fitted to each of the curves and these are shown in bold. The equations for each of the "fitted" polynomials appears to the right of the respective curve, along with its "goodness of fit".

$a x^{3}+b x^{2}+c x+d$, rather than $a x^{2}+b x+c$. The rate of mutation acquisition is at first slow and increasing, up to a threshold point; after this point, the cell continues to gain mutations, but the rate of acquisition decreases. If this theory is in fact correct, then it would follow that the first two curves, are in fact passed the threshold point at time $t=0$. The turning point at which the mutation acquisition rate goes from increasing to decreasing occurs at some point $t<0$ and hence does not appear. Since the second two curves $\left(m_{1}=0.2\right.$ and $\left.m_{1}=0.5\right)$ still have an increasing rate of mutation acquisition, it would suggest that this "threshold" point has not yet been reached. If we were to continue to observe the behavior of the two curves $m_{1}=0.2$ and $m_{1}=0.5$, then, they would reach their "threshold point" and the rate of mutation acquisition would decrease until the entire genome was mutated (i.e. $\bar{I}_{x}(t)=100$ ).

To test this theory, we considered the case $m=0.05, m_{1}=0.5$ and followed the evolution of the cell population through 200 cell cycles. The resultant plot is shown in Figure 3. As can be seen from the plot, the function for this curve is a cubic polynomial (with "goodness of fit" $R^{2}=0.9984$ ), which supports our theory that the rate of mutation would continue increasing to a point and then begin to decrease. Differentiating this and solving for $\bar{I}_{x}(t)=0$ will give the cell cycle at which the turning point occurs.

We repeated this procedure, this time keeping the repair rate constant at $m_{1}=0.05$ and considering mutation rates $m=0.05, m=0.01$ and $m=0.5$. The mean phenotype was then plotted against time $t$ (in cell cycles), for the three different mutation rates considered (Figure 4). Once again, we see large rates of mutation acquisition at the beginning, which decrease with time. Once the entire population has a fully mutated genome, the individuals in the population can no longer acquire mutations and consequently the mutation acquisition rate will be zero.

The curves representing the mean phenotype of the population, may be better expressed using the logistic differential equation. ${ }^{19}$ This model is based on the assumption that a population grows at a rate proportional 

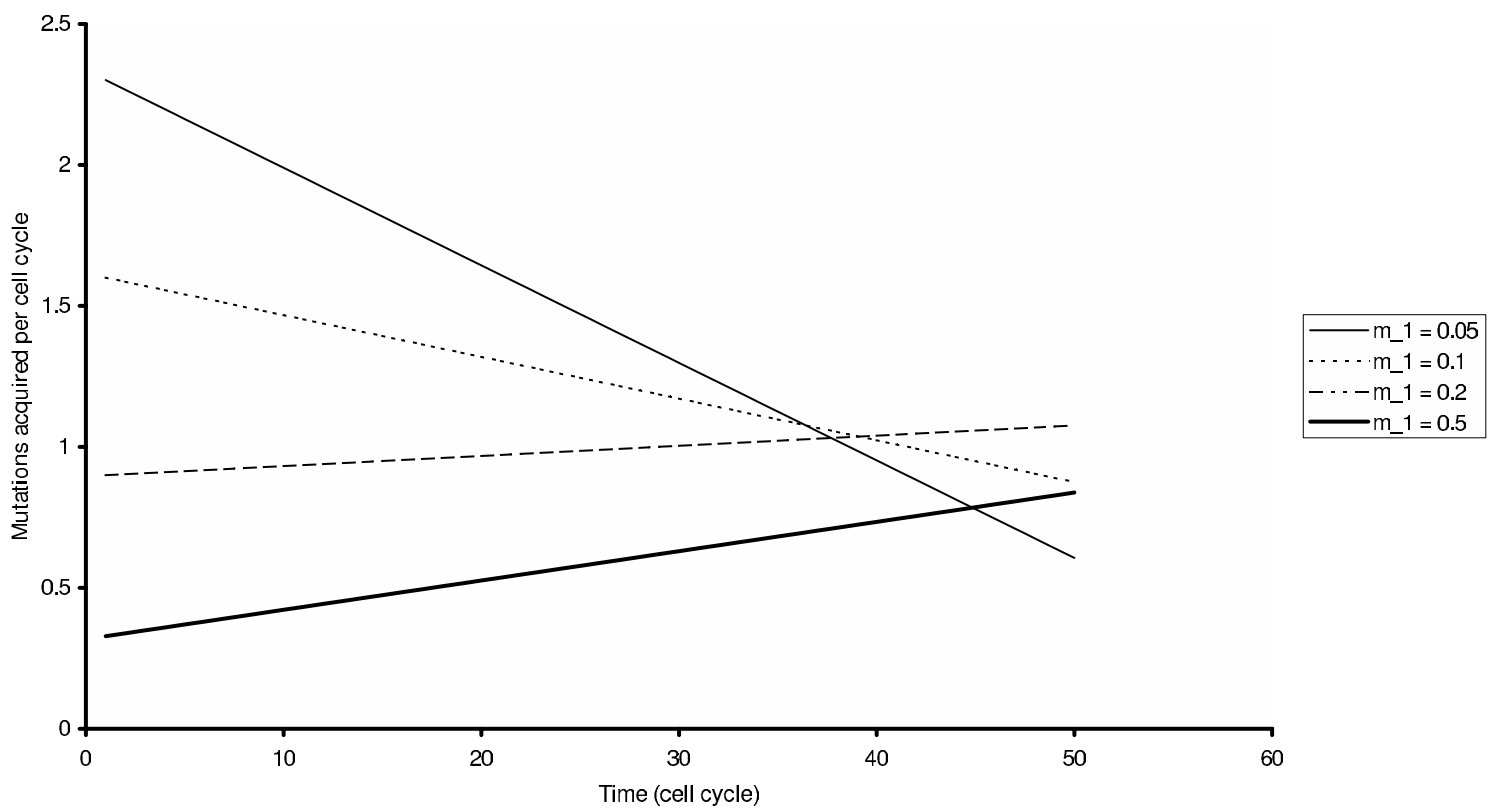

Figure 2. Rate of mutation acquisition per cell cycle. Mutation rate $m=0.05$. Repair rates considered: $m_{1}=0.05$ $m_{1}=0.1 m_{1}=0.2$ and $m_{1}=0.5$. The equations from Figure 1, for the fitted polynomials, were differentiated with respect to $t$ and plotted. Each line shown, displays the mean rate of mutation acquisition at time $t$ for the respective repair rates considered.

to the the size of the population and is expressed as:

$$
\frac{d \bar{I}}{d t}=k \bar{I}\left(1-\frac{\bar{I}}{K},\right.
$$

where: $\bar{I}$ is the mean phenotype/number of mutations,

$k$ is the proportionality constant and

$K$ is the carrying capacity.

For our model, the carrying capacity $K$ would be 100 , (the length of the the genome $G$ ) and the proportionality constant $k$ would be determined by the ratio between the mutation and repair rates, ( $m$ and $m_{1}$ respectively).

\section{DISCUSSION}

From the results obtained, it was suggested that the mean phenotype $\bar{I}_{x}(t)$ with respect to time is determined by a cubic polynomial of $t$. The rate of mutation acquisition increases to a point, before decreasing as the mean phenotype of the progeny approaches a maximum.

In healthy cells, the acquisition of mutations is kept in check by the repair mechanisms of the cell. This is best represented by the curve in Figure 1 where the rate of repair is ten times the rate of mutation $\left(m_{1}=0.5\right.$, $m=0.05$ ). There is a greater latency in obtaining mutations, when compared to all the other cases (i.e. a significantly lower rate of mutation acquisition). Over time, however more and more mutations are acquired and the repair mechanisms of the cell become less efficient, allowing the rate at which these mutations are acquired to increase, up to a point. Past this point, as the cell becomes more and more mutated, it is no longer able to sustain this increasing rate of mutation. Consequently, this rate drops off, until the entire genome is mutated.

Cells in our body behave in a similar manner. Over time, they acquire mutations, which are ordinarily kept in check by repair mechanisms, such as those initiated by the p53 gene. As cells age, however, the efficiency 


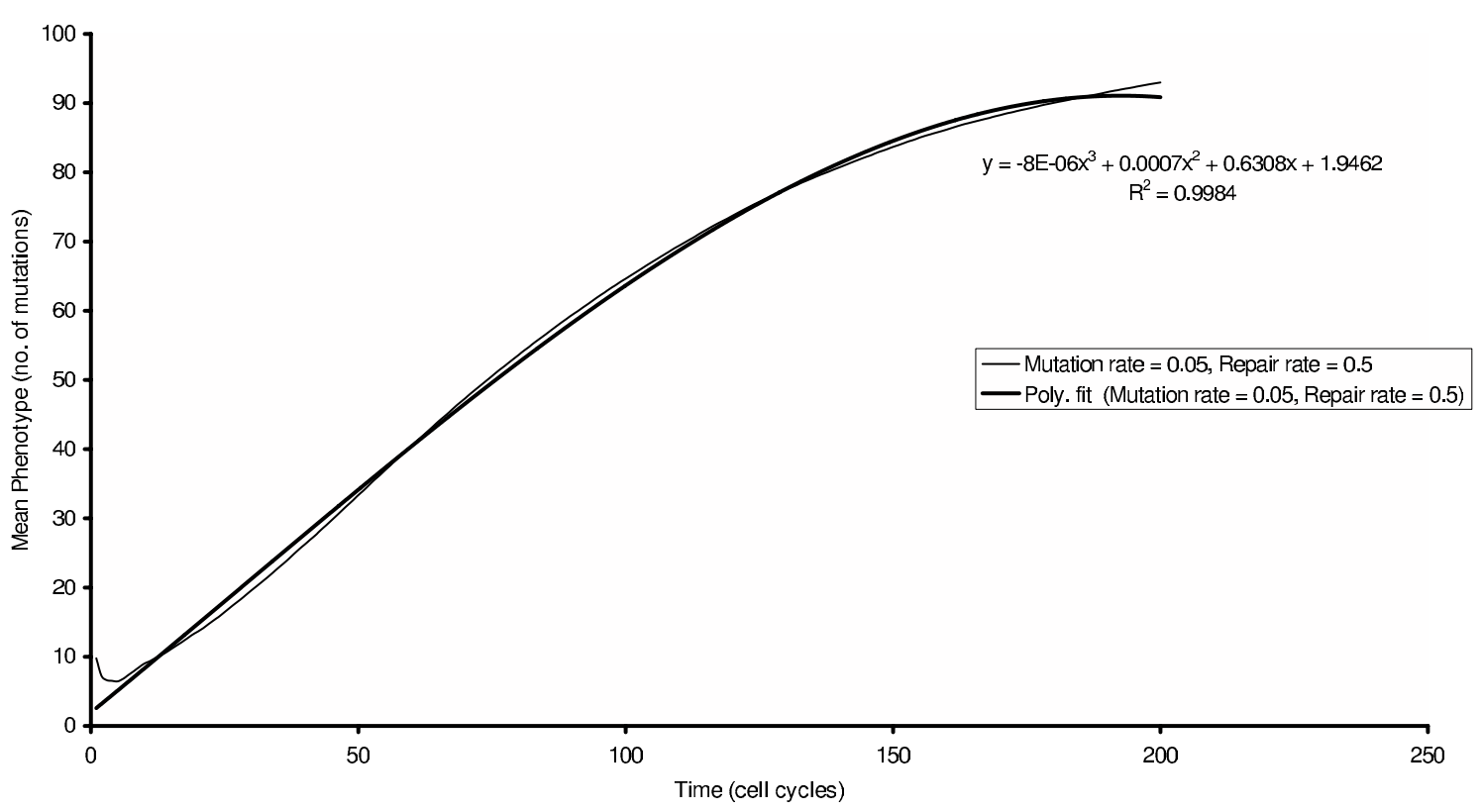

Figure 3. The genomic activity of the case with mutation rate $m=0.05$ and repair rate $m_{1}=0.5$ was observed over 200 cell cycles. The actual curve is the fine line and the bold line over the top is the fitted polynomial. The equation for the polynomial is shown, with its "goodness of fit" value. Watching the evolution over a longer time period, showed that the shape of the curve changes from convex to concave. The point of inflection is the "threshold point" where the rate of mutation acquisition changes from increasing to decreasing.

of the repair mechanisms decrease and the cell develops more and more mutations. As the mutations in a cell accumulate, the cell can become malignant, and a tumor will result. We see in Figure 4, with the mutation rate ten times the repair rate, the repair rate of the cell has virtually no impact on keeping the mutation rate in check and the progeny of cells accumulate a mean phenotype of 100 after only 10 cell cycles.

Future research planned involves using more realistic mutation and repair rates, and the interactions of gene and drug therapies on these, to determine their usefulness and efficacy. Another interesting question is, given the mutations in p53, what are the benefits and drawbacks in using radiotherapy, which while killing off some cells will further mutate others?

\section{CONCLUSION}

Our model offers a simplistic view of the effects of mutation on a population of cells. The model considers the role of p53 in maintaining genomic integrity, and investigates how mutations to this gene affect the evolution of a population of cells. We see that the repair mechanisms of the cell are not able to prevent mutations of the cell indefinitely, but instead increase the latency of mutation acquisition. Because the efficiency of the repair mechanism in cells decreases with age, all replicating cells are predisposed to potentially developing into a tumor with further mutations fixed in upon replication. ${ }^{20}$ Methods that can prolong the time that mutation of the cell is kept in check will reduce the potential of tumor development. The results show that increasing the ratio between the repair and mutation rates increases the ability of the cell to keep mutations in check for longer. Any future gene therapies or drug treatments could potentially alter these rates in order to prolong life or possibly even prevent tumors progressing through to cancer.

\section{ACKNOWLEDGMENTS}

Useful discussions with Cosma Shalizi, University of Michigan, and David Krakauer, Santa Fe Institute, are acknowledged. 


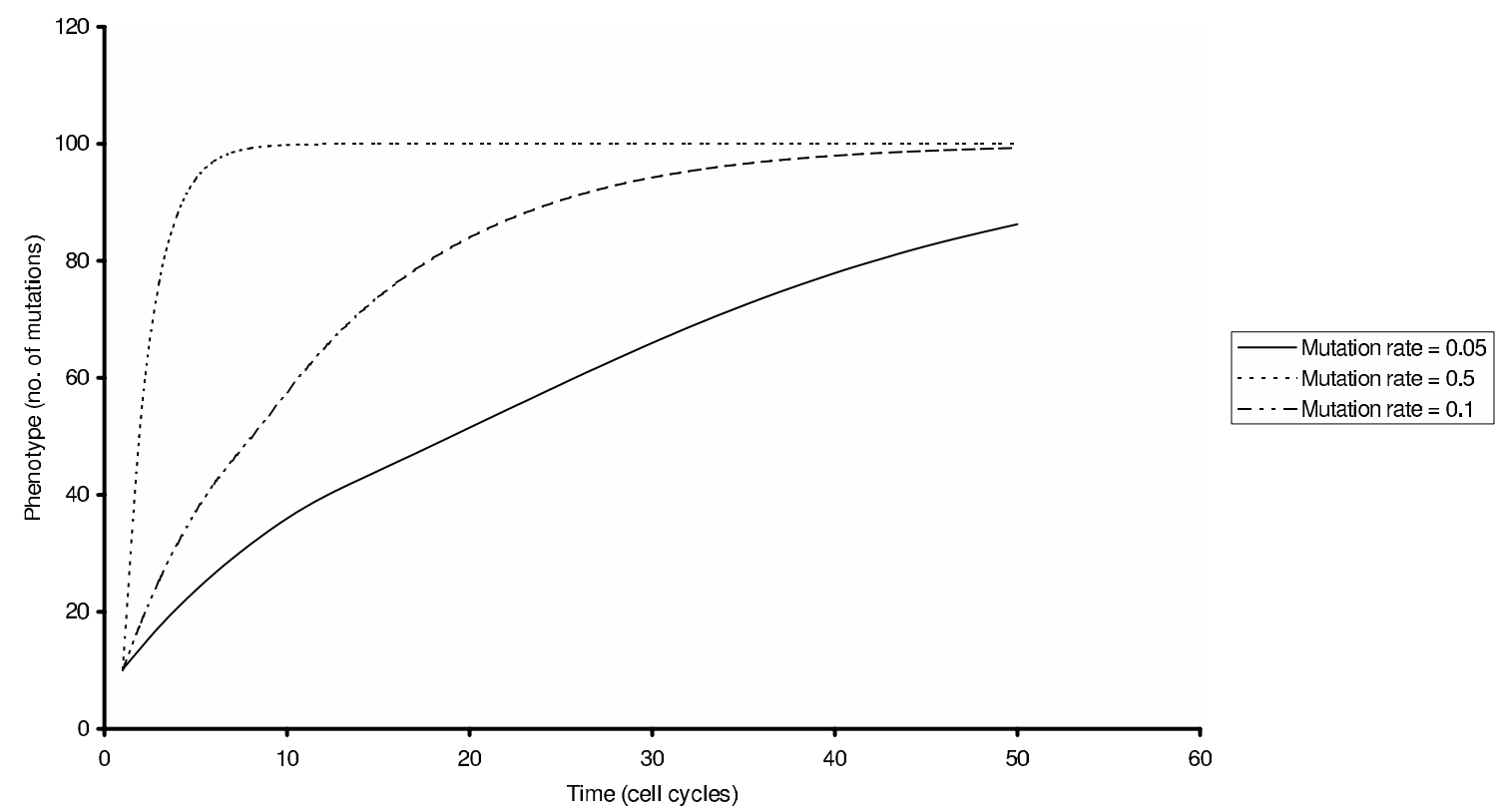

Figure 4. Mean phenotype (number of mutations) of cells against generations. Repair rate $m=0.05$. Mutation rates considered: $m=0.05 m=0.1$ and $m=0.5$. The concave shape of the curves indicates that the rate of mutation acquisition is decreasing, and hence the inflection points would have occurred at time $t<0$. For all curves, mutations are acquired at a decreasing rate, until a maximum phenotype of 100 is obtained.

\section{REFERENCES}

1. T. Caspari, "Checkpoints: how to activate p53," Curr. Bio. 10(8), pp. R315-R317, 2000.

2. D. Gold and D. Sokolowski, "p53 protein-DNA complex," http://biology.kenyon.edu/courses/biol64/ chime20003/p53/FRAMES/captxt.htm, 2004.

3. B. A. Montelone, "Mutation, mutagens \& DNA repair," http://www-personal.ksu.edu/ bethmont/mutdes.html, 1998.

4. W. H. Elliott and E. D. C., Biochemistry and Molecular Biology, Oxford University Press, 2nd ed., 1997.

5. F. Janus, N. Albrechtsen, I. Dornreiter, L. Wiesmuller, F. Grosse, and W. Deppert, "The dual role model for p53 in maintaining genomic integrity," Cell Mol. Life Sci. 55(1), pp. 12-27, 1999.

6. B. B. Zhou and S. J. Elledge, "The DNA damage response: putting checkpoints in perspective," Nature 408, pp. 433-439, 2000.

7. R. R. Wallace-Brodeur and S. W. Lowe, "Clinical implications of p53 mutations," Cell Mol. Life Sci. 55, pp. 64-75, 1999.

8. B. Vogelstein, D. Lane, and A. J. Levine, "Surfing the p53 network," Nature 408, pp. 307-310, 2000.

9. C. J. Sherr, "Tumor surveillance via the ARF-p53 pathway," Genes and Development 12(19), pp. 2984-2991, 1998.

10. S. J. Elledge, "Cell cycle checkpoints: preventing an identity crisis," Science 274, pp. 1664-1672, 1996.

11. N. D. Lakin and S. P. Jackson, "Regulation of p53 in response to DNA damage," Oncogene 18(54), pp. 7644$7655,1999$.

12. B. Alberts, A. Johnson, J. Lewis, M. Raff, K. Roberts, and P. Walter, Molecular Biology of the Cell, Garland Science, 4th ed., 2002.

13. R. D. Wood, M. Mitchel, J. Sgouros, and T. Lindahl, "Human DNA repair genes," Science 291(5507), pp. 1284-1289, 2001. 
14. J. Yuan, T. M. Yeasky, P. A. Havre, and P. M. Glazer, "Induction of p53 in mouse cells decreases mutagenesis by UV radiation," Carcinogenesis 16, pp. 2295-2300, 1995.

15. D. Goukassian, F. Gad, M. Yaar, M. S. Eller, U. S. Nehal, and G. B. A., "Mechanisms and implications of the age associated decrease in DNA repair capacity," The Faseb Journal 14, pp. 1325-1334, 2000.

16. A. J. F. Griffths, J. H. Miller, D. T. Suzuki, R. C. Lewontin, and W. M. Gelbart, An Introduction to Genetic Analysis, W. H. Freeman \& Company, 6th ed., 1996.

17. S. W. Lowe and A. W. Lin, "Apoptosis in cancer," Carcinogenesis 21(3), pp. 485-495, 2000.

18. L. D. Attardi and T. Jacks, "The role of p53 in tumor supression: lessons from mouse models," Cell Mol. Life. Sci. 55(1), pp. 48-63, 1999.

19. N. Boccara, Modeling Complex Systems, Springer-Verlag, 2003.

20. S. L. Spencer, M. J. Berryman, J. García, and D. Abbott, "An ordinary differential equation model for the multistep transformation to cancer," Journal of Theoretical Biology 231, pp. 515-524, 2004. 